\title{
Targeted Genome Editing for Crop Improvement
}

\author{
Hyeran $\mathrm{Kim}^{1 \dagger}$, Sang-Tae $\mathrm{Kim}^{1 \dagger}$, Sang-Gyu Kim${ }^{1} *$, Jin-Soo $\mathrm{Kim}^{1,2 *}$ \\ ${ }^{1}$ Center for Genome Engineering, Institute for Basic Science, Yuseong-gu, Daejeon 34047, Korea \\ ${ }^{2}$ Department of Chemistry, Seoul National University, Seoul 08826, Korea
}

\begin{abstract}
Crop improvement is essential to attaining world food security and enhancing nutrition for human beings. Both conventional breeding and modern molecular breeding have contributed to increased crop production and quality. However, the time and resources for breeding practices have been limited. It takes a long time to bring a novel improved crop to the market, and the genetic sources from wild species cannot be always available for crops of our interests. Genome editing technology implemented molecular breeding can overcome those limitations of time and resource by facilitating the specific editing of plant genomes. However, there is a long-lasting argument about the safety of genetically modified organisms (GMOs). In this review, we briefly summarize the principle of genome editing tools, focusing on the CRISPR/Cas9 system and the application of these tools to plants in the service of crop engineering.
\end{abstract}

Keywords Cas9, CRISPR, Crop improvement, Genome editing, GMO, Molecular breeding

\section{Principle of CRISPR/Cas9 system}

The recent development of genome editing technology using programmable nucleases such as zinc finger nucleases (ZFNs); transcription activator-like effector nucleases (TALENs); clustered regularly interspaced short palindromic repeats (CRISPR) and CRISPR-associated (Cas) proteins (CRISPR/Cas) (Kim and Kim 2014) shed light on a new plant breeding approach; this technique can minimize the degree to which the target genome is genetically modified and can increase the specificity of the target locus (Shan et al. 2013; Araki \& Ishii 2015; Baltes and Voytas 2015; Kanchiswamy et al. 2015). CRISPR/Cas9, in particular, originated from the bacterial immune system of Streptococcus pyogenes and has been intensively introduced since seminal reports suggested relatively simple RNAguided nuclease systems might be more broadly applied to numerous biological organisms (Deltcheva et al. 2011; Jinek et al. 2012).

These genome editing technologies use the cell's endogenous repair system when specific genomic regions are manipulated using sequence-specific nucleases (SSNs). SSNs can drive double-strand breaks (DSBs) in targeted sites of genomic DNA; those DSBs are repaired by processes known as non-homologous end-joining (NHEJ) (Rouet et al. 1994) and homology-directed repair (HDR) (Bibikova et al. 2002) (Fig. 1). During the NHEJ repair process, various types of error such as the insertion or deletion of nucleotides by imperfect ligation at the tip of DSB points may be generated. In contrast, because the HDR pathway uses a template for repairing DSBs, the process of the HDR is more precise than that of NHEJ. In plants, however, the NHEJ process is far superior to the HDR process probably because a replaceable template DNA is often located in determinant cells in which HDR is not the preferred repair mechanism (Knoll et al. 2014).

The first- and second-generation system of SSNs, ZFNs and TALENs, are dependent upon proteins' ability to recognize specific DNA sites and nuclease activity of FokI domains to cleave the target sequences (Kim and Kim 2014). ZFN is composed of 3-6 zinc finger proteins (ZFPs),

Received October 30, 2015; Revised November 22, 2015; Accepted November 23, 2015; Published November 30, 2015

*Comesponding author Jin-Soo Kim, jskim01@snu.ac.kr, Tel: +82-2-880-9327, Fax: +82-2-874-7455

*Comesponding author Sang-Gyu Kim, sgkim@ibs.re.kr, Tel: +82-42-878-8301, Fax: +82-42-878-8399

${ }^{\dagger}$ These authors contributed equally to this work. 


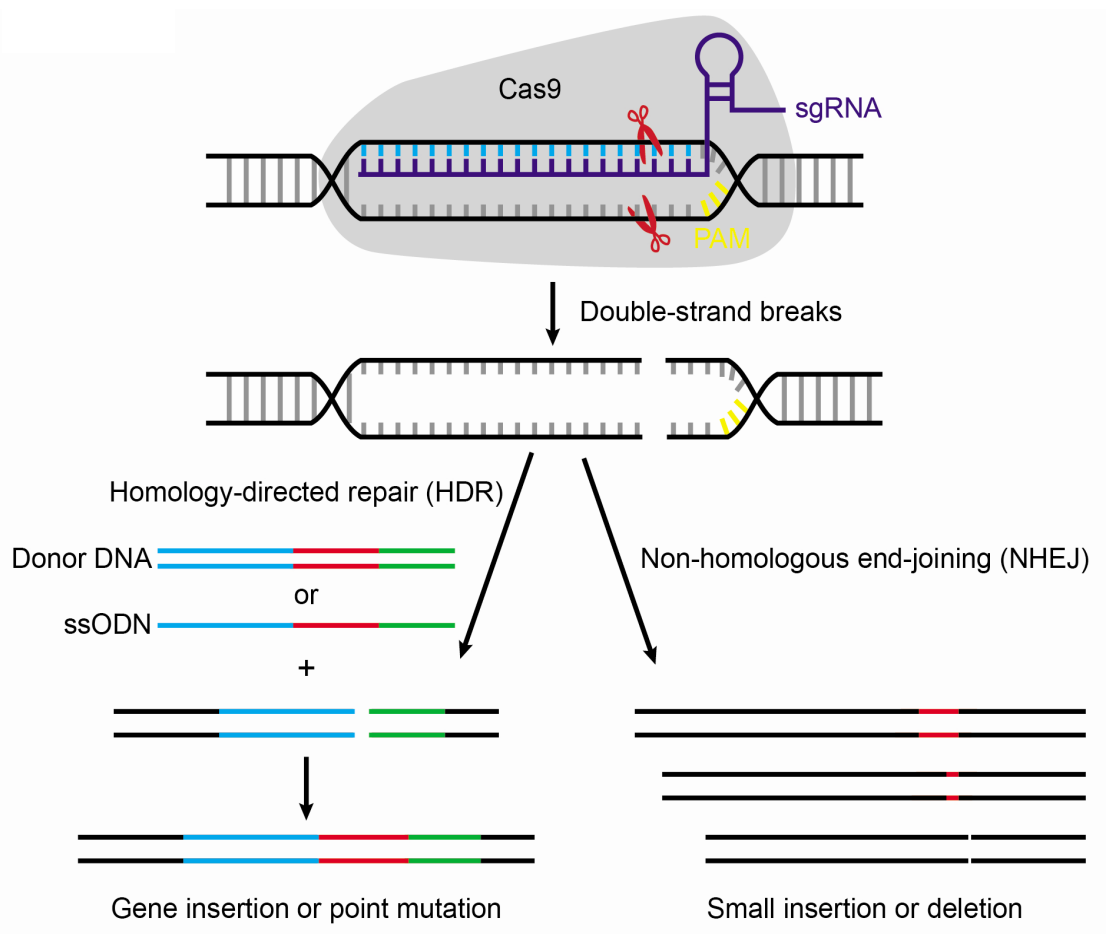

Fig. 1. CRISPR/Cas9 system for targeted genome editing. Cas9 and single guide RNA (sgRNA) complex can recognize a specific site in genome guided by a short sequence (19-20 bp) in sgRNA. Two endonuclease domains within Cas9 protein cleave double-stranded DNA 3bp upstream of the protospacer adjacent motif (PAM, 5'-NGG-3' for Streptococcus pyogenes Cas9). These DNA double strand breaks (DSBs) can facilitate genome editing through homology-directed repair (HDR) with donor DNA or single strand oligodeoxynucleotide (ssODN) or error-prone non-homologous end-joining (NHEJ) repair pathway.

and each of these proteins can recognize three base pairs of target DNA sequence. Two FokI nuclease domains attached to two subsets of ZFPs perform the DSBs. Like ZFNs, the TALEN system is composed of TALE DNA binding proteins, and each protein can recognize a specific single DNA base pair. Two FokI nuclease domains are attached to two subsets of TALE proteins to carry out DSBs at the target site.

More simply, the third-generation SSN system, CRISPR/ Cas9, uses one protein for nuclease activity and a short single-strand guide RNA (sgRNA) sequence to guide this protein to the target sites (Fig. 1). In the bacteria's immune system, captured small DNA fragments ( $20 \mathrm{bp}$ ) from the foreign DNA of invading phages or plasmids are kept in the bacteria's own genome; these fragments, known as protospacers, form a CRISPR (Makarova et al. 2006; Barrangou et al. 2007). In type II CRISPR systems, these CRISPR regions are transcribed and processed into target-specific CRISPR RNA (crNRA). For this process, common trans-activating crRNA (tracrRNA) is needed. These crRNA and tracrRNA are combined with Cas9 protein to form a dual RNA-Cas9 complex; this complex recognizes the 19-20 nucleotide sequences in the target genome matching to those in crRNA complementarily (Deltcheva et al. 2011). An sgRNA containing essential portions of crRNA and tracrRNA is often used as a convenient form (Jinek et al. 2012; Fig. 1). To cleave target DNA, Cas9 derived from $S$. pyogenes (SpCas9) first recognizes the protospacer-adjacent motif (PAM), 5'-NGG-3' in the target site (Mojica et al. 2009) and binds the target sequence guided by sgRNA or crRNA, which hybridizes with 19-20 bp DNA sequences upstream of the PAM. SpCas9 then cleaves target DNA at 3 bp upstream of the PAM (Jinek et al. 2012).

For more detailed information about genome editing tools and comparisons of these tools, readers are encouraged to 
visit the brief history of CRISPR/Cas system (Doudna and Charpentier 2014; Hsu et al. 2014), and the reviews of its mechanism and information about comparisons (Kim and Kim 2014; Boettcher and McManus 2015; Sternberg and Doudna 2015).

\section{Crop improvement using genome-editing techniques}

One of the major goals of conventional plant breeding is to remove or add certain traits of crops to enhance their nutritional values or resistance to diverse biotic and abiotic stress (Allard 1999; Moose and Mumm 2008). For instance, the high level of erucic acids and glucosinolates in rapeseed was successfully removed by conventional breeding in 1970s, and now rapeseed has become the third most important source of vegetable oils in the world (Gupta and Pratap 2007). While this conventional breeding relies chiefly on natural variation in a gene of interest, physical or chemical mutagens have been used to generate random crop variants. In addition, the development of RNAi methods enables target genes to be silenced in specific tissues or at certain times, which results in the removal of unwanted traits from crops (Kusaba 2004; Tang and Galili 2004). However, RNAi-mediated gene silencing has had to overcome challenges from incomplete gene silencing, the co-silencing of unintended genes (off-targets), and the random integration of foreign DNA into plant genomes (if T-DNA harboring RNAi construct is transformed). Genome editing technologies can overcome some of these limitations of conventional breeding and RNAi-based approach to be used to generate improved crops indistinguishable from naturally occurring mutant crops.

Since the successful genome editing using CRISPR/Cas9 system in Arabidopsis (Feng et al. 2013; Li et al. 2013) and rice (Miao et al. 2013; Shan et al. 2013) has been reported, many independent applications of this genome-editing tool in various crops have shown that CRISPR/Cas9 system is broadly acceptable and effective for crop improvement (e.g. sweet orange, Jia and Wang 2014; maize, Liang et al. 2014; tomato, Brooks et al. 2014; Svitashev et al. 2015; soybean, Jacobs et al. 2015; Li et al. 2015; Sun et al. 2015).
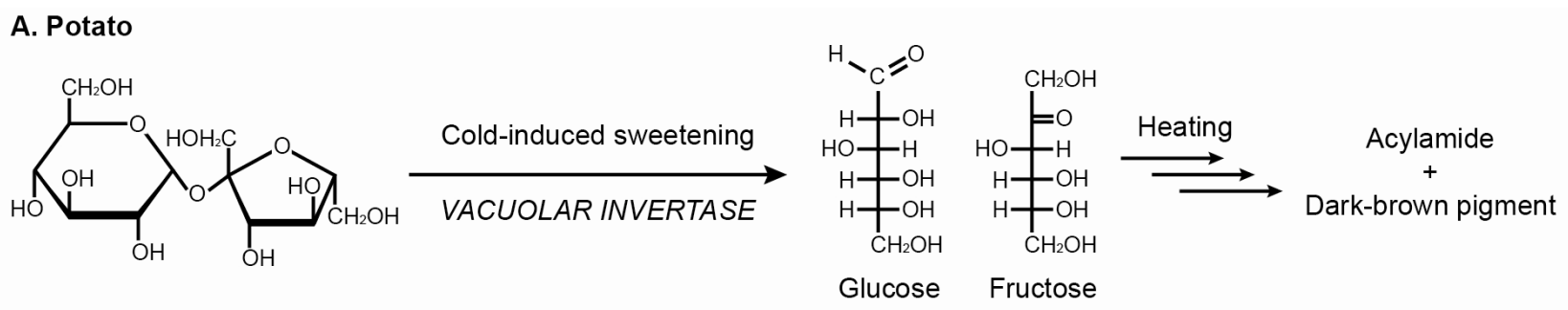

\section{B. Apple}<smiles>[R]c1ccc(O)c(O)c1</smiles>

Exposure to oxygen after cutting

POLYPHENOL OXIDASE

Polyphenol

\section{Maize}

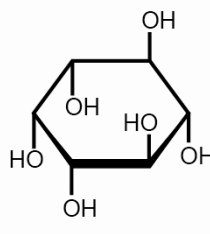

myo-Inositol

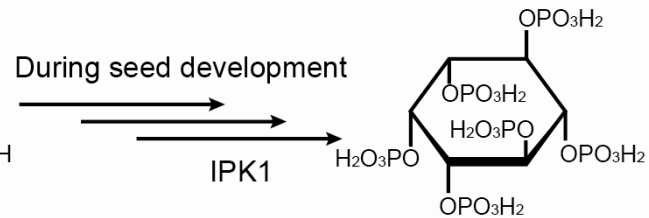

Phytic acid

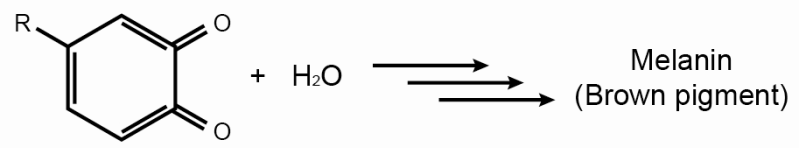

Quinone

D. Coffee
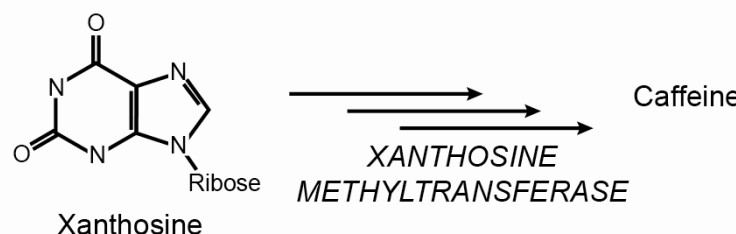

METHYLTRANSFERASE

Fig. 2. Examples of potential target genes for crop improvement. Genome editing tools will be widely used to remove unnecessary chemicals in crops, such as (A) acrylamide in potato, (B) melanin in apple, (C) phytic acid in maize, and (D) caffeine in coffee. There is a list of genome-edited plants by ZFN, TALEN, or CRISPR/Cas9 system (Baltes and Voytas 2014; Araki and IshiI 2015). 
The technical issues, such as enhancing the chance of germline transmission from genome-edited mutant lines (Hyun et al. 2014; Mao et al. 2015; Yan et al. 2015) or DNA-free gene editing (Woo et al. 2015), have been examined recently. We describe here several examples of how genome-editing tools can contribute to crop improvement.

\section{Acrylamide-free potatoes}

Potato are normally harvested once a year and stored in a cold chamber to keep them fresh and prevent sprouting before cooking. During cold storage, starch in the potato tuber is degraded into glucose and fructose (Fig. 2A). Unfortunately, this cold-induced sweetening causes serious problems when potatoes are turned into potato chips or French fries; at high temperatures, the reduced sugars turn into dark-brown pigments and a strong carcinogen, acrylamide, forms during this process. Using a technique that relied on RNAi, the JR Simplot company developed the "innate potato" (Chawla et al. 2012), and this creation was approved by U.S. Food and Drug Administration (FDA) in 2015. In addition, Voytas and his colleagues recently designed TALEN constructs to mutate VASCULAR INVERTASE genes, which convert sucrose to glucose and fructose in a potato tuber; they showed that processing genome-edited potatoes at high temperatures after cold storage produced fewer brown pigments and acrylamides compared to wild-type potatoes (Clasen et al. 2015).

\section{Non-browning apples}

Browning in fresh-cut apples and apple juice is linked to the enzymatic reaction mediated by POLYPHENOL OXYDASE (PPO) genes. A short time after an apple is cut, polyphenol compounds inside it lose hydrogen (because the flesh of the apple is oxidized by PPOs), and a brown pigment appears, melanin (Fig. 2B). The Canadian company Okanagan Specialty Fruits ${ }^{\mathrm{TM}}$ transformed apples with an RNAi construct to silence four PPO genes in apples and successfully generated non-browning apples known as 'Arctic apple'. This GM apple was approved by the US Department of Agriculture (USDA) and will be on the market soon (Waltz 2015b). The RNAi technique was also used to silence homologous genes in potatoes to reduce browning (Waltz 2015a). The CRISPR/Cas system can be used to mutate $P P O$ genes and generate non-browning apples or potatoes that contain no foreign DNA.

\section{Low phytic acid in maize}

Maize (corn) contains high levels of phosphorous, but most of phosphorous are stored in the form of phytic acid that is poorly digested in human. Mutant lines containing low levels of phytic acid were isolated by conventional breeding and mutagenesis, but it has proven difficult to introduce these mutations into different accessions of maize (Raboy 2007). Again, the RNAi technique has been used to silence the expression of phytic acid biosynthesis genes or transporter genes in maize (Raboy 2007). In 2009, Shukla et al. designed ZFN constructs to mutate the IPK1 gene, one of the phytic acid biosynthesis genes (Fig. 2C), and this genome-edited maize is undergoing tests with the hope that it will soon be on the market.

\section{Coffee without Caffeine}

Finding caffeine-free coffee is the long-term goal of coffee breeders (Borrell 2012), because the process of removing caffeine from normal coffee beans usually costs a lot, sometimes produces toxic byproducts, and may reduce or remove other flavors. In 2003, Ogita et al. designed RNAi constructs to silence the caffeine biosynthetic gene, XANTHOSINE METHYLTRANSFERASE in Coffea canephora, known as Rubusta coffee (Fig. 2D). However, it should be mentioned that these 'non-caffeine' coffee trees did not produce beans, because floral organ development was desynchronized in the transgenic lines (Borrell 2012). Other caffeine biosynthetic genes or caffeine transporters will be the future target of genome editing, as scientists try to make a caffeine-free coffee.

\section{Herbicide-resistant crops}

Genome editing techniques can generate targeted point mutations in crops. One of the earliest studies, ZFNmediated genome editing, directed specific DNA sequences into the target locus and introduced a point mutation in the ACETOLACTATE SYNTHASE (ALS) gene, which is the target of sulfonylurea (SU) and imidazolinone (IMI) herbicides (Townsend et al. 2009). Long-term use of these 


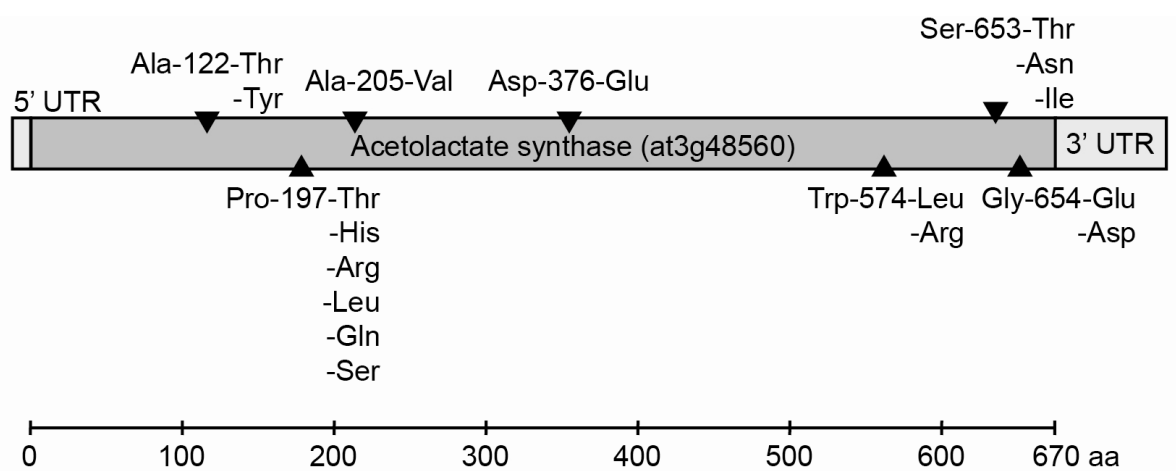

Fig. 3. Amino acid substitutions in acetolactate synthase confer resistance to ALS-inhibiting herbicides. Acetolactate synthase (ALS) is the key enzyme in the biosynthesis of valine, leucine, and isoleucine. ALS enzyme activity is inhibited by the treatment of sulfonylurea (SU) or imidazolinone (IMI), which are the major herbicides used worldwide to control weeds. Long-term treatment of these herbicides has forced to evolve herbicide-resistant weeds, which mainly have amino acid changes in the ALS enzyme. Single amino acid substitution confers the resistance to SU or IMI.

herbicides has selected for herbicide-resistant weeds, which have a site-specific mutation mainly on the ALS gene (Fig. 3) (Powles and Yu 2010). Using our knowledge of genetic variants from the environment, the point mutation can be introduced at the target site by genome editing technologies to generate herbicide-resistant crops. These genome-edited plants could potentially be exempted from GMO regulation, if no foreign DNA remains in edited plants.

\section{Durable disease resistance crops via CRISPR/Cas9 mediated knock-out}

Two research groups simultaneously proposed a possible strategy to make plants virus resistant using CRISPR/Cas9 technology (Baltes et al. 2015; Ji et al. 2015). Both have shown that the Cas9/sgRNA expressed in plant cells effectively interfered with the viral replication and generated geminivirus-resistant Nicotiana benthamiana and Arabidopsis thaliana as CRISPR/Cas9 system originally confers the resistance of bacteria against virus attacks. In addition, the application of CRISPR/Cas9 could extend to a direct knock-out strategy in the diseasesusceptible genes, often termed "S-genes," of a host genome; the result is the development of durable disease-resistant crops. Ever since the barley $M L O$ gene was revealed to be responsible for susceptibility to powdery mildew fungi (Büschges et al. 1997), this locus was a popular target of genome engineering for scientists hoping to confer broad-spectrum resistance to powdery mildew in barley and wheat (Büschges et al. 1997; Várallyay et al. 2012). More recently, the S-gene knockout strategy was employed successfully in hexaploid bread wheat using the TALEN and CRISPR/Cas9 system, and the fungi-resistant wheat was generated (Wang et al. 2014).

\section{Desired trait knock-in via CRISPR systems}

A new RNA-guided genome engineering tool, the CRISPR/Cpf1 system, was reported to have properties different from those of the CRISPR/Cas9 system: the CRISPR/Cpf1 system has a single RNA-guided endonuclease lacking tracrRNA, 5' T-rich PAM, and a 5-nt staggered DNA cut (Zetsche et al. 2015). If Cpf1 exhibits the same properties, especially the "staggered DNA cut" in the plant system, the CRISPR/Cpf1 technique could be rapidly adapted to gene conversion; moreover, the technique allows for an efficient, targeted gene insertion unlike the homology arm-directed recombination. Thus the CRISPR/Cpf1 together with the CRISPR/Cas9 system could immensely accelerate the targeted gene knock-in strategy for crop improvement.

\section{DNA-free genome-edited crops via CRISPR/Cas9 nibonucleoprotein (RNP)}

As the CRISPR/Cas9 system consists simply of an 
sgRNA and a Cas9 protein, it can be assembled by in vitro transcribed sgRNA together with a recombinant Cas9 protein without introducing a plasmid encoding the sgRNA and Cas9 protein in a cell. In vitro pre-assembled CRISPR/Cas9 RNP complexes were delivered successfully to mammalian cells and plant cells (Kim et al. 2014; Woo et al. 2015). Even a single protoplast-driven edited lettuce was successfully regenerated into a whole plant and subsequently transmitted the edited alleles to offspring (Woo et al. 2015). Although gene delivery of the plasmidmediated expression system is stable, it is still possible to integrate the fragmentation of plasmids into the plant genome. In contrast, RNP delivery has no chance to leave a fragment of foreign DNA into the target genome during transmission (Kim et al. 2014). Moreover, RNP delivery directly introduces all active components -- Cas9 and sgRNA complexes -- into target cells; as RNP complexes are subsequently degraded within a short period of time, achieved the concise and elaborate editing through the reduction of off-target effects (Kim et al. 2014; Woo et al. 2015). Since the CRISPR/Cas9 RNP strategy successfully generated genome-edited lettuce without any foreign DNA integration, it may become a promising technique for improving crops.

\section{Perspectives for plant breeding}

Recent advances in genome engineering have led us to a new era: crop genome editing will bring about the next "green revolution." The CRISPR/Cas9 technique has led scientists and breeders to develop new strategies for crop improvement, which could provide sustainable solutions for the global food crisis. By domesticating wild plants, we have been able to generate high-yield crops and to produce staple foods. Besides of the high-yield production and staple foods development, we have met two concerns; both global environmental changes and reduced genetic diversity in ecological system. The new genome editing techniques with RNP-based transformation could pave the way for solving problems in food security, developing cultivars by which desired traits from a gene pool of wild species. Thus, novel and valuable plants generated by genome editing techniques can regain useful traits overlooked during domestication; these traits help plants survive unpredictable global environmental changes.

\section{ACKNOWLEDGEMENTS}

This work is supported by Institute for Basic Science (IBS-R021-D1). We thank Emily Wheeler, Boston, for editorial assistance.

\section{REFERENCES}

Allard RW. 1999. Principles of plant breeding. 2nd ed. John Wiley \& Sons, New York.

Araki M, Ishii T. 2015. Towards social acceptance of plant breeding by genome editing. Trends Plant Sci. 20: 1-5.

Baltes NJ, Hummel AW, Konecna E, Cegan R, Bruns AN, Bisaro DM, Voytas DF. 2015. Conferring resistance to geminiviruses with the CRISPR-Cas prokaryotic immune system. Nat. Plants 1: 15145.

Baltes NJ, Voytas DF. 2015. Enabling plant synthetic biology through genome engineering. Trends Biotechnol. 33: 120-131.

Barrangou R, Fremaux C, Deveau H, Richards M, Boyaval P, Moineau S, Romero DA, Horvath, P. 2007. CRISPR provides acquired resistance against viruses in prokaryotes. Science 315: 1709-1712.

Bibikova M, Golic M, Golic KG, Carroll D. 2002. Targeted chromosomal cleavage and mutagenesis in Drosophila using zinc-finger nucleases. Genetics 161: 1169-1175.

Boettcher M, McManus MT. 2015. Choosing the right tool for the job: RNAi, TALEN, or CRISPR. Mol. Cell 58: 575-585.

Borrell B. 2012. Plant biotechnology: Make it a decaf. Nat. News 483: 264-266.

Brooks C, Nekrasov V, Lippman ZB, Eck JV. 2014. Efficient gene editing in tomato in the first generation using the clustered regularly interspaced short palindromic repeats/ CRISPR-associated9 system. Plant Physiol. 166: 12921297.

Büschges R, Hollricher K, Panstruga R, Simons G, Wolter M, Frijters A, van Daelen R, van der Lee T, Diergaarde P, Groenendijk J, et al. 1997. The barley Mlo gene: a novel control element of plant pathogen resistance. Cell 88: 
695705.

Chawla R, Shakya R, Rommens CM. 2012. Tuber-specific silencing of asparagine synthetase-1 reduces the acrylamide-forming potential of potatoes grown in the field without affecting tuber shape and yield. Plant Biotechnol. J. 10: 913-924.

Clasen BM, Stoddard TJ, Luo S, Demorest ZL, Li J, Cedrone F, Tibebu R, Davison S, Ray EE, Daulhac A, et al. 2015. Improving cold storage and processing traits in potato through targeted gene knockout. Plant Biotechnol. J. (in press).

Deltcheva E, Chylinski K, Sharma CM, Gonzales K, Chao Y, Pirzada ZA, Eckert MR, Vogel J, Charpentier E. 2011. CRISPR RNA maturation by trans-encoded small RNA and host factor RNase III. Nature 471: 602-607.

Doudna JA, Charpentier E. 2014. The new frontier of genome engineering with CRISPR-Cas9. Science 346: 1258096.

Feng Z, Zhang B, Ding W, Liu X, Yang D, Wei P, Cao F, Zhu S, Zhang F, Mao Y, Zhu J. 2013. Efficient genome editing in plants using a CRISPR/Cas system. Cell Res. 23: $1229-1232$.

Gupta SK, Pratap A. 2007. History, origin, and evolution. Adv. Bot. Res. 45: 1-20.

Hsu PD, Lander ES, Zhang F. 2014. Development and applications of CRISPR-Cas9 for genome engineering. Cell 157: 1262-1278.

Jacobs TB, LaFayette PR, Schmitz RJ, Parrott WA. 2015. Targeted genome modifications in soybean with CRISPR/Cas9. BMC Biotechnol. 15: 16.

Ji X, Zhang H, Zhang Y, Wang Y, Gao C. 2015. Establishing a CRISPR-Cas-like immune system conferring DNA virus resistance in plants. Nat. Plants 1: 15144.

Jia H, Wang N. 2014. Targeted genome editing of sweet orange using Cas9/sgRNA. PLoS ONE 9(4): e93806.

Jinek M, Chylinski K, Fonfara I, Hauer M, Doudna JA, Charpentier E. 2012. A programmable dual-RNA-guided DNA endonuclease in adaptive bacterial immunity. Science 337: 816-821.

Kanchiswamy CN, Malnoy M, Velasco R, Kim JS, Viola R. 2015. Non-GMO genetically edited crop plants. Trends Biotechnol. 33: 489-491.

Kim H, Kim JS. 2014. A guide to genome engineering with programmable nucleases. Nat. Rev. Genet. 15: 321-34.

Kim S, Kim D, Cho SW, Kim J, Kim JS. 2014. Highly efficient RNA-guided genome editing in human cells via delivery of purified Cas9 ribonucleoproteins. Genome Res. 24: 1012-1019.

Knoll A, Fauser F, Puchta H. 2014. DNA recombination in somatic plant cells: mechanisms and evolutionary consequences. Chromosome Res. 22: 191-201.

Kusaba M. 2004. RNA interference in crop plants. Curr. Opin. Biotechnol. 15: 139-143.

Li J, Norville JE, Aach J, McCormack M, Zhang D, Bush J, Church GM, Sheen J. 2013. Multiplex and homologous recombination-mediated genome editing in Arabidopsis and Nicotiana benthamiana using guide RNA and Cas9. Nat. Biotechnol. 31: 688-691.

Li Z, Liu Z, Xing A, Moon BP, Koellhoffer JP, Huang L, Ward RT, Clifton E, Falco SC, Cigan AM. 2015. Cas9-guide RNA directed genome editing in soybean. Plant Physiol. 169: 960-970.

Liang Z, Zhang K, Chen K, Gao C. 2014. Targeted mutagenesis in Zea mays using TALENs and the CRISPR/Cas system. J. Genet. Genomics 41: 63-68.

Makarova KS, Grishin N, Shabalina SA, Wolf YI, Koonin EV. 2006. A putative RNA-interference-based immune system in prokaryotes: computational analysis of the predicted enzymatic machinery, functional analogies with eukaryotic RNAi, and hypothetical mechanisms of action. Biol. Direct 1: 7 .

Mao Y, Zhang Z, Feng Z, Wei P, Zhang H, Botella JR, Zhu JK. 2015. Development of germ-line-specific CRISPRCas9 systems to improve the production of heritable gene modifications in Arabidopsis. Plant. Biotechnol. J. (in press).

Miao J, Guo D, Zhang J, Huang Q, Qin G, Zhang X, Wan J, $\mathrm{Gu}$ H, Qu L. 2013. Targeted mutagenesis in rice using CRISPR-Cas system. Cell Res. 23: 1233-1236.

Mojica FJM, Diez-Villasenor C, Garcia-Martinez J, Almendros C. 2009. Short motif sequences determine the targets of the prokaryotic CRISPR defence system. Microbiol. 155(3): 733-740.

Moose SP, Mumm RH. 2008. Molecular plant breeding as the foundation for 21st century crop improvement. Plant Physiol. 147: 969-977.

Ogita S, Uefuji H, Yamaguchi Y, Koizumi N, Sano H. 2003. RNA interference: producing decaffeinated coffee plants. Nature 423: 823-823.

Powles SB, Yu Q. 2010. Evolution in action: plants resistant to herbicides. Annual Rev. Plant Biol. 61: 317-347. 
Raboy V. 2007. The ABCs of low-phytate crops. Nat. Biotechnol. 25: 874-875.

Rouet P, Smih F, Jasin M. 1994. Introduction of doublestrand breaks into the genome of mouse cells by expression of a rare-cutting endonuclease. Mol. Cell Biol. 14: 8096-8106.

Shan Q, Wang Y, Li J, Zhang Y, Chen K, Liang Z, Zhang K, Liu J, Xi JJ, Qiu JL, et al. 2013. Targeted genome modification of crop plants using a CRISPR-Cas system. Nat. Biotechnol. 31: 686-688.

Shukla VK, Doyon Y, Miller JC, DeKelver RC, Moehle EA, Worden SE, Mitchell JC, Arnold NL, Gopalan S, Meng $\mathrm{X}$, et al. 2009. Precise genome modification in the crop species Zea mays using zinc-finger nucleases. Nature 459: 437-441.

Sternberg SH, Doudna JA. 2015. Expanding the biologist's toolkit with CRISPR-Cas9. Mol. Cell 58: 568-574.

Sun X, Hu Z, Chen R, Jiang Q, Song G, Zhang H, Xi Y. 2015. Targeted mutagenesis in soybean using the CRISPR-Cas9 system. Sci. Rep. 5: 10342.

Svitashev S, Young JK, Schwartz C, Gao H, Falco SC, Cigan AM. 2015. Targeted mutagenesis, precise gene editing, and site-specific gene insertion in Maize using Cas9 and guide RNA. Plant Physiol. 169(2): 931-945.

Tang G, Galili G. 2004. Using RNAi to improve plant nutritional value: from mechanism to application. Trends Biotechnol. 22: 463-469.

Townsend JA, Wright DA, Winfrey RJ, Fu F, Maeder ML,
Joung JK, Voytas DF. 2009. High-frequency modification of plant genes using engineered zinc-finger nucleases. Nature 459: 442-445.

Várallyay É, Giczey G, Burgyán J. 2012. Virus-induced gene silencing of Mlo genes induces powdery mildew resistance in Triticum aestivum. Arch. Virol. 157: 1345-1350.

Waltz E. 2015a. USDA approves next-generation GM potato. Nat. Biotechnol. 33: 12-13.

Waltz E. 2015b. Nonbrowning GM apple cleared for market. Nat. Biotechnol. 33: 326-327.

Wang Y, Cheng X, Shan Q, Zhang Y, Liu J, Gao C, Qiu J-L. 2014. Simultaneous editing of three homoeoalleles in hexaploid bread wheat confers heritable resistance to powdery mildew. Nat. Biotechnol. 32: 947-951.

Woo JW, Kim J, Kwon S, Corvalán C, Cho SW, Kim H, Kim SG, Kim ST, Choe S, Kim JS. 2015. DNA-free genome editing in plants with preassembled CRISPR-Cas9 ribonucleoproteins, Nat. Biotechnol. 33: 1162-1164.

Yan L, Wei S, Wu Y, Hu R, Li H, Yang W, Xie Q. 2015. High efficiency genome editing in Arabidopsis using Yao promoter-driven CRISPR/Cas9 system. Mol. Plant. (in press).

Zetsche B, Gootenberg JS, Abudayyeh OO, Slaymaker IM, Makarova KS, Essletzbichler P, Volz SE, Joung J, van der Oost J, Regev A, et al. 2015. Cpf1 is a single RNA-guided endonuclease of a class 2 CRISPR-Cas system. Cell. (in press). 